\title{
1 A review of the dental caries status of ethnic minority children in
}

2 China

3

4

5 Abstract

6

China has 55 ethnic minority groups comprised of 113 million persons, or $7.0 \%$ of total population. Dental caries is a major health problem for children in China, and national oral health surveys currently report dental caries based on geographical location rather than by ethnic group. This study reviews the literature on dental caries in ethnic minority children in China. Publications were retrieved in Chinese and English from five electronic databases; thirty-eight studies from 1983 to 2012 met inclusion criteria and described 25 ethnic minority groups. Primary dentition median caries prevalence and experience were higher (51\% and $d m f t=3.0$, respectively) than permanent dentition caries prevalence and experience $(39 \%$ and DMFT=0.8). Median caries prevalence was highest (80\%) for permanent dentition among aggregated ethnic minorities with population greater than 1 million. More work and research is needed to expand dental caries prevention and treatment measures for ethnic minority child populations in China. 


\section{Introduction}

China is a large East Asian country with an area of 9.6 million square kilometers. The total population of mainland China is approximately 1.4 billion and is the largest in the world. Its populace comprises 56 ethnic groups [1]. Han is the main ethnic group, composed of approximately $93 \%$ of the population. The populations of other ethnic minority groups vary from a few thousand to more than 16 million. These ethnic groups have diverse languages, religions and cultures (Table 1) and are widely scattered across the country (Figure 1). Some live in the more developed eastern or central regions such as Manchu, but the majority live in the less developed mountainous inland or border districts in the western region [2]. The average per capita gross domestic product (GDP) in ethnic minority districts in 2010 was about US\$3500 [3], which was about $73 \%$ of the national GDP (US\$4800) [4]. To reduce the adverse effects of this large population on the developing country, the central government implemented the one child policy in the 1970 s to slow down the population growth. However, this one-child policy does not apply to ethnic minority groups. As a result, the population growth rate of the ethnic minorities in China has increased over the past two decades and reached 6.9\% in 2010 [1]. The ethnic minority population was approximately 113 million in 2010 , which is greater than the population of any country in the European Union [1].

Race/ethnicity has been identified as a factor for oral health status. Many studies conducted overseas suggest that people from specific ethnic minorities often have poor oral health status and increased risk of dental decay $[5,6]$. While socio-economic status is one of the most important factors in dental inequalities, underlying cultural beliefs and practices can influence oral health status through diet, dental care-seeking behavior, or the use of home remedies [7], which are fundamental in framing appropriate oral health policies and the development of effective oral health services and oral health promotion activities [8].

General health care for children is always among the first priorities of national 
health policies, and oral health is an integral part of general health [9]. Poor dentition significantly affects children's nutrition, and consequently their growth, development and general health. Dental caries is a major health problem for children and has become a huge burden in China. Dental caries causes pain and infection and advanced caries will progress into the tooth pulp and eventually form a dental abscess [10]. In the Philippines, the main reason for absence from school was dental caries [11] and an estimated 51,000,000 school hours were missed among children in the US in 2000 [12]. In China, three national oral health surveys were performed in 1983, 1995 and 2005 $[13,14]$. Their findings indicate that although the prevalence of dental caries and caries experience among children in China has declined during the past two decades, dental caries is still a significant health problem affecting many children [14]. The three national oral health surveys document the oral health of children geographically according to province, but ethnicity is not a routinely collected category.

Determining the oral health of children is important for planning and implementing services oriented toward meeting the needs of the population [15]. The oral health of children can also be used to predict adult oral health and oral health needs [16]. It is important to understand the dental caries status in a multicultural society with different social development to ensure effective delivery of interventions and optimal allocation of resources. A literature search found no reviews of this imperative issue in China. This paper reports a literature review of the studies on dental caries in ethnic minority children in China.

\section{Materials and Methods}

\section{Search strategy}

To identify epidemiological studies on the ethnic minorities in China, a broad search of publications was conducted to include as many relevant publications as possible. The search was performed using a Chinese database (China Academic Journals Full-text Database) and two English electronic databases (PubMed, ISI Web 
of Science) between January 1980 and December 2012. Another two databases of Chinese theses (China Master's Theses Full-text Database, China Doctor Dissertations Full-text Database) from 1984-2012 were also included in the search (Figure 2). The key words used for searching were (Chinese and English as appropriate) as follows: (dental caries OR caries OR decay) AND (ethnic minority OR ethnic groups) AND (China or Chinese) AND (child OR children OR adolescent OR student). If the publications contained the search thesaurus, they were selected to generate a list of potentially eligible studies to be included in this review.

\section{Study selection}

A manual search was carried out on the list of potentially eligible studies. The selected publications were screened by title and abstract. Duplicated reports or studies using the same data were excluded. Publications were included if they fulfilled the following inclusion criteria: a) examining one or more ethnic minority groups; b) assessing the primary teeth of children aged 3 to 6 years, or assessing the permanent teeth of children aged 11 to 14 years, and c) reporting caries prevalence or caries experience. Caries prevalence in \% reports the proportion of the population who is suffering from (untreated) dental decay; whereas caries experience in number of teeth reports the proportion of the population who suffered (treated) or is suffering from dental decayed. The caries experience of primary and permanent teeth are expressed in dmft and DMFT scores, respectively. The dmft and DMFT measure the sum of the number of decayed teeth (dt or DT), missing teeth due to decay (mt or MT) and filled teeth ( $\mathrm{ft}$ or FT) in a child. Publications that reported the combined caries prevalence or combined mean caries experience DMFT and dmft scores or combined caries prevalence of primary and permanent dentition were excluded.

\section{Synthesis of data}

The full papers of the selected publications were obtained. The bibliographies of the publications deemed eligible were searched manually and additional relevant publications were included in the review. The results of these studies were grouped into 
two groups according to the age of the survey children: 3 to 6 years old for primary dentition and 11 to 14 years old for permanent dentition. A summary was made for each publication, according to a) ethnic group, b) investigators and year of publication, c) survey site, d) children's age range, e) sample size, f) sampling method, and g) dental caries prevalence and experience.

\section{Results}

The initial search identified 175 reports in the five databases (Figure 2). Duplicate reports in different databases were not counted. Most of the selected publications were found in the China Journals Full-text Database $(\mathrm{N}=120)$ and PubMed $(\mathrm{N}=43)$. These 175 publications were screened manually by abstract and title. Seventeen studies were found to be duplications of another study and thus were excluded. Seventy-nine reports were found to be unrelated to reporting of dental caries status among children of ethnic minority groups in China and they were also excluded. Consequently, 79 publications were identified, and their full papers were obtained. A further 24 publications were identified from the bibliographies and were included in the review. However, 65 publications were excluded. The majority of the studies $(52 / 65)$ were excluded because the age of the sample was not mentioned or the caries assessment was on mixed dentition, or not specific to primary or permanent dentition. Thirteen studies reported the caries status of at least two ethnic groups or the status of Han and other ethnic groups. Finally, 38 publications were included in this literature review.

All 38 publications were cross-sectional studies published between 1983 and 2012. Two studies were published in the 1980s, 15 in the 1990s and 21 from 2000 onward. These studies evaluated the dental caries status of children from 25 ethnic minorities. More than half $(14 / 25)$ of the studies referred to ethnic groups with populations of more than 1 million. There were 20 ethnic groups among the 3 - to 6year-old children included in studies of primary dentition (Table 2). The median caries 
prevalence and caries experience in $\mathrm{dmft}$ for these children were $51 \%$ and 3.0 , respectively. There were 23 ethnic groups among the 11- to 14-year-old children included in studies of permanent dentition. The median caries prevalence and caries experience in DMFT for 11- to 14-year-old children were 39\% and 0.8. The findings according to ethnic group are summarized in Table 3 for primary dentition and in Table 4 for permanent dentition.

\section{Primary dentition}

The Korean children living in Jilin province in central China had the highest caries prevalence (94\%) in their primary teeth (Table 3), whereas Mongolian children living in Qinghai province in northwestern China had the lowest prevalence (11\%). The highest caries experience in primary teeth was found in Bonan children living in northwestern China $(\mathrm{dmft}=7.5)$ and Bulang children living in southwestern China had the lowest caries experience $(\mathrm{dmft}=0.1)$. Only one study reported decayed teeth $(\mathrm{dt})$, missing teeth due to caries (mt) and filled teeth (ft) [17]. Six studies mentioned that most of the decayed teeth were left untreated [17-22].

Three studies reported the dental caries experience according to gender $[18,23$, 24]. None of them found significant gender difference [18, 23, 24]. Five surveys compared the dental caries status of ethnic minority children with that of Han children. Zhuang children living in western China had higher dmft scores than Han children (3.4 vs 2.7) [17]. Their frequent bottle feeding habit with sugary products was attributed as one of the main reasons for the high caries prevalence. Higher caries prevalence was also found in Uygur children in northwestern China than in Han children (67\% vs 54\%) [26]. Three studies reported that Hui (33\% vs 95\%), Tibetan (46\% vs $81 \%$ ), Uygur (39\% vs 90\%) and Yi children (36\% vs 95\%) had a lower prevalence of dental caries than that of Han children [24, 27, 46]

\section{Permanent dentition}

The highest caries prevalence in permanent teeth (84\%) was found among 11- 
to 14-year-old Tibetan children in Tibet province in northwestern China, whereas the lowest prevalence (7\%) was found in Tujia children in Hubei province in central China (Table 4). The highest caries experience in mean DMFT was 2.3 among the Naxi children living in Yunnan province in southwestern China [28]. Bulang children in Yunnan province had the lowest caries experience, and their mean DMFT was 0.1 [29]. Two studies reported that girls had higher caries prevalence than boys in Dongxiang (26\% vs $18 \%$ ) and Kirgiz children (73\% vs $65 \%$ ) [25,48]. One study found the caries prevalence and caries experience to be higher in Tibetan children than in Han children (44\% vs 24\%) [32]. Another study found that the caries prevalence of Dongxiang (41\%), Bonan (44\%) and Yugu children (46\%) living in northwestern China was higher than that of Han children (19\%) living in the same province [33].

\section{Discussion}

The national oral health surveys in China report dental caries according to geographic location. As the Han population dominates in majority of the provinces [4], reporting the caries status by province does not reflect the dental caries status of ethnic minorities in China. Dental caries is a multifactor disease and the possible roles of socio-economic and cultural factors have been highlighted [34]. The ethnic minority population in China is more than 110 million, thus it is essential to study dental caries according to ethnic group. This is the first article to review the literature on dental caries in ethnic minority children in China. The sources used in this review were thought to be exhaustive. They are regarded to be major databases that include the most prominent biomedical studies. The source used also contains supplemental information which published in non-medical journals. Databases in Chinese were also used in this review that include not only journal articles in Chinese but also most if not all of the master's and doctoral theses published by all dental schools and universities in China. The great majority of the studies on the caries status of children in China were assumed to be published in either Chinese or English. 
This review shows that studies on the oral health of ethnic minority children in

China are generally disorganized and sporadic. The total number of publications is small and covers less than half of the ethnic minority groups. There was no dental caries information on children from 30 ethnic minorities: Achang, Buyei, Daur, Deang, Derung, Dong, Erwenki, Gaoshan, Gelao, Hazak, Hezhen, Jing, Lhoba, Manchu, Monan, Monba, Mulao, Nu, Oroqen, Pumi, Qiang, Russ, Salar, She, Shui, Tajik, Tatar, Va, Wuzibieke and Xibe.

The caries status of children is a major indictor of oral health [35]. The World Health Organization (WHO) selected 5- and 12-year-old children as the indicator age groups for assessing the caries status of primary and permanent dentition in children [35]. This should help to standardize the data collected and allows comparison of the dental caries status of primary and permanent dentition between studies. However, more than half of the publications identified did not follow the recommendation. Many studies reported the combined caries status of primary and permanent dentition and hence could not be included in the review. This study included surveys reporting the dental caries status of children aged 3 to 6 for primary teeth, with the aim of including more publications in the literature search. Children younger than 3 were not included as their teeth might not have erupted. Children older than 6 were not included because their primary teeth might have exfoliated and were not available for assessment. An extended age range of 11 to 14 years old was adopted for searching the caries status of permanent dentition. Children younger than 11 would probably be in the mixed dentition stage and by 14 the second molar should have erupted for assessment. Some surveys collectively reported the caries status of children of more than one ethnic group, perhaps due to small sample sizes. The results of these studies were not included in the review.

The majority of the studies $(34 / 38)$ basically used the dental caries diagnosis criteria recommended by WHO. However, many studies (23/38) did not provide details of the sampling methods and none reported the sample size estimation, which is 
essential for making inferences about the studied population. In addition, most publications (34/38) did not mention the training and calibration of the examiners, the number of examiners and the agreement between their dental caries assessments. Therefore, the validity and reliability of examiners in diagnosing the dental caries could be problematic. Only two studies duplicated the examinations and reported the interexaminer agreement $[17,32]$. It is essential to develop a standardized survey method to ensure the repeatability, comparability and validity of the results obtained by different studies. The training and calibration of examiners is important to achieve good validity and reproducibility.

Few studies were performed on children of ethnic minorities with populations of less than 1 million. However, the prevalence and experience of caries in primary teeth was higher in these ethnic groups. For example, the caries prevalence of Dongxiang children was 79\% [20] and Naxi was 71\% [28]. The caries status of primary and permanent dentition varied substantially between ethnic groups in the same region $[19,20]$. One study reported that children of an ethnic group living in isolated areas had little decay [36]. The study found $75 \%$ of the 5-year-olds Lahu children and $85 \%$ of the 12-year-olds had no caries experience.

No consistent conclusions could be drawn from studies comparing the dental caries status of the ethnic minority groups and Han children. Only one study conducted a multifactorial analysis and found that the caries experience of children was associated with bottle feeding in babyhood and ethnicity [17]. Future studies should examine the relationship between dental caries status and potential risk factors such as language barriers, religion, oral health beliefs etc. Most studies found no significant difference between the dental caries status of boys and girls in primary dentition. This finding is similar to those of the second and third national surveys [13, 14]. A higher prevalence of caries in the permanent dentition of children was found in girls. This finding also concurs with that of the national surveys and most local surveys on Han children. Moreover, it is known that green tea, white tea, black tea, pu-er tea, oolong tea etc. are 
largely consumed by adults in China. Many different types of tea contain substantial amount of fluoride but its consumption by children is unknown. Further studies should be carried out to investigate the drinking of tea by children and its effect on dental caries.

In general, studies conducted after 2000 reported a higher caries experience in ethnic minority children than the figure reported by the national oral health survey. This is consistent with the increased consumption of sugar and inadequate exposure to professional fluoride application [37]. Basic dental hygiene education such as twice daily tooth brushing and use of fluoride toothpaste, toothbrushes and floss are very important in ethnic minority children. The children should have access to these items, and should be educated in schools or at home. This prevention is one of the most important steps especially in low resource areas that cannot support municipal fluoridation and qualified dentists. The caries experiences in the primary dentition of ethnic minority children reported in the 1990s were all lower than those of the second national oral health survey in 1995. Nevertheless, some studies published after 2000 reported a higher caries experience in ethnic minority children than in Han children [19, 20, 22-24]. Two studies conducted in the 1990s reported the caries experience of permanent teeth in Yao, Lisu and Lahu children was higher than that reported in the second national survey $[29,38]$. Several studies [20, 25, 30, 31, 33] reported the caries experience of permanent teeth in Bonan, Dongxiang, Krigiz, Korean, Li, Naxi and Yugur children was higher than that reported in the third nation oral health survey in 2005 [14]. The oral health promotion program and school-based dental sealant placement program target large cities or affluent areas in China [39]. There are no organized caries prevention programs for children living in rural areas in China [40]. The ethnic minority children have limited access to dental care services and resources because most of them, particularly those with low populations, live in isolated rural areas.

Only one study [17] reported the percentage of untreated decayed teeth (dt), although many studies commented that most decayed teeth were left untreated [20, 25, 
$28,30,31,33,41]$. The dentist to population ratio has been reported to be around 1:100,000 [42]. The great shortage of dentists in China, especially in rural areas, is likely to be one of the main reasons for the lack of treatment. Treating the massive amount of caries in children in these areas is challenging. The cost of conventional dental treatment and the shortage of dental health care workers make it difficult to manage caries in children [42]. Treatment that slows down or ceases caries progression is a pragmatic way of minimizing children's discomfort and problems due to caries. The provincial governments should perform epidemiological surveys to identify communities with higher caries prevalence and allocate resources to prevent and control dental caries among children. Reporting the oral health of people in mainland China according to their ethnic minority group in future national oral health surveys would also be helpful. This information would help to identify and address the unique oral health needs of culturally diverse children. The government and professional societies should also attract dentists to work in areas where ethnic minorities live. Dental training should be provided so that simple dental treatments such as topical fluoride applications and atraumatic restorative treatment can be performed to control caries among children. In the long term, oral health promotion strategies including water fluoridation should be considered to promote the quality of oral health among these children.

\section{Conclusions}

There are 55 ethnic minorities with a total population of more than 110 million living in predominantly rural areas of China. Dental caries is the one of the major health problems for children and has become a huge burden in China. The past three national surveys reported dental diseases based on geographical location but not ethnic group. Ethnicity is likely to be an important factor in dental caries. Various ethnic groups have different cultures and life styles, which can be important confounding factors affecting the distribution of dental caries among children. An epidemiological survey based on ethnic groups is crucial for planning, implementing and monitoring community programs to improve the oral health of children in China. Although oral health surveys 
316 have been conducted on 25 of the minority groups, the survey methods of these studies

317 were not standardized, thus comparison between studies is difficult. More studies with 318 appropriate design should be carried out to identify high risk groups for early 319 intervention.

320 


\section{References}

322 1. National Bureau of Statistics of China. The 2010 statistical report on the 323 national population $2013 \quad\left[\begin{array}{ll}\ln & \text { Chinese }] \text {. }\end{array}\right.$ 324 http://www.stats.gov.cn/tjfx/jdfx/t20110428_402722253.htm. Date last accessed 8 July 3252013.

326 2. Gustafsson BA, Sai D. Temporary and persistent poverty among ethnic 327 minorities and the majority in rural China. Review of Income and Wealth 2009; $328 \quad 55(\mathrm{~s} 1): 588-606$.

329 3. LE CH, Sheng LY. China's ethnic statistical yearbook [In Chinese]. Peking, 330 China: China Statistics Press; 2011.

331 4. Sheng LY. China statistical yearbook [In Chinese]. Peking, China: China 332 Statistics Press; 2011.

333 5. Gray M, Morris AJ, Davies J. The oral health of South Asian five-year-old children in deprived areas of Dudley compared with White children of equal deprivation and fluoridation status. Community Dent Health 2000; 17(4):243-5.

6. Pine CM, Adair PM, Nicoll AD, Burnside G, Petersen PE, et al,. International comparisons of health inequalities in childhood dental caries. Community Dent Health 2004; 21(1 Suppl):121-30.

339 7. Riedy CA, Weinstein P, Milgrom P, Bruss M. An ethnographic study for understanding children's oral health in a multicultural community. Int Dent J 2001; 51(4):305-12.

342 8. Marino R, Morgan M, Hopcraft M. Transcultural dental training: addressing the oral health care needs of people from culturally diverse backgrounds. Community Dent 344 Oral Epidemiol 2012;40(Suppl 2):134-40.

9. World Health Organization. The liverpool declaration: promoting oral health in the 21st century 2013.http://www.who.int/oral_health/events/liverpool_declaration/en/. Date last accessed 8 July, 2013.

348 10. Chu $\mathrm{CH}$. Treatment of early childhood caries: a review and case report. Gen Dent 2000; 48(2):142-8. 
11. Department of Education, Health and Nutrition Center. National oral health survey among the public school population in the Philippines, 2006. Manila, Philippines; 2008.

12. Beaglehole R, Benzian H, Mackay J. The oral health atlas. UK: FDI World Dental Federation; 2009.

13. Wang HY, Petersen PE, Bian JY, Zhang BX. The second national survey of oral health status of children and adults in China. Int Dent J 2002; 52(4):283-90.

14. Qi XQ. Report of the third national survey of oral health. Peking, China: People's Medical Publishing House; 2008.

15. Chu CH, Chau AM, Lo EC, Lam A. Planning and implementation of community oral health programs for caries management in children. Gen Dent 2012; 60(3):210-7. 16. Pearce MS, Steele JG, Mason J, Walls AW, Parker L. Do circumstances in early life contribute to tooth retention in middle age? J Dent Res 2004; 83(7):562-6.

17. Zeng X, Luo Y, Du M, Bedi R. Dental caries experience of preschool children from different ethnic groups in Guangxi Province in China. Oral Health Prev Dent 2005; $3(1): 25-31$.

18. Chen J. Oral health survey among 388 Han and Zang children in Qinghai [In Chinese]. Qinghai Medical Journal 2006; 36(6):70-1.

19. Chen Y, Li ZQ, Nie HB, Zhou HJ, Liu WJ. Investigation of dental caries of 5year-old children and their parents' oral hygienic knowledge and behavior in Dongxiang, Baoan and Yugu nationalities [In Chinese]. Journal of Lanzhou University (Medical Sciences) 2009; 35(2):27-30.

20. Dong H. Eidemiological investigation of the caries of the Dongxiang, Yugu, Baoan minority people [In Chinese]. Oral Medicine, Lanzhou University; 2007.

21. Li DH. Dental caries status among preschool children in Liangshan, Sichuan Province [In Chinese]. West China Journal of Stomatology 1998; 13(1):70-1.

22. Ma LY, Lei T, Li XL, Zhou HJ, Li ZQ. Dental caries among Tibetan children in Gannan reign [In Chinese]. Chinese Journal of Conservative Dentistry 2009; 19(7):428. 23. Fang XH. Investigation of dental caries status of Korean preschool children [In Chinese]. Journal of Clinical Stomatology 2001; 17(1):78. 
24. Huang C, Wen Y, Ye Y, Ren XQ. Epidemiological characteristics and prevention and control effects of caries in preschool children [In Chinese]. Journal of Kunming Medical Universtiy 2012; 33(7):114-7.

25. Xuehereti Y. Reporting of dental caries status among Kirgiz school children in Kezilesu region, Xinjiang Provinace [In Chinese]. Journal of Modern Stomatology 2004; 18(5):470-1.

26. Nurbiye M. Epidemiological investigation on deciduous caries of 3-to-5-yearold Uygur and Chinese children in Urumqi [In Chinese]. Stomatology 2011; 31(8):48896.

27. Ayiguli T, Asiya Y. A survey of the dental caries status among 450 Uygur and Han ethnic preschool children [In Chinese]. Chinese Journal of Child Health Care 2004; 12(5):457.

28. Liu J, Zhang CH, Liu XR, Li YH, Yu B, et al,. The logistic regression analysis of dental caries status and related factors among Naxi minority children [In Chinese]. Journal of Kunming Medical University 2009; 30(9):19-22.

29. Zhang QK, Yuan CY, Li CW. Oral health survey on three ethnic minorities children in Monghai County, Yunnan Province [In Chinese]. Shanghai Journal of Stomatology 1994; 3(1):46-7.

30. Liu ZM. Research of oral health status and its related factors of Korean children in Yanji City [In Chinese]. University of Yanbian; 2008.

31. Wu HX, He ST. Caries survey of $1680 \mathrm{Li}$ ethnic minority primary school students in Sanya [In Chinese]. Journal of Hainan Medical University 2010; 16(3):3516.

32. Lo EC, Jin LJ, Zee KY, Leung WK, Corbet EF. Oral health status and treatment need of 11-13-year-old urban children in Tibet, China. Community Dent Health 2000; 17(3):161-4.

33. Zhou HJ, Nie HB, Ma LY, Fu SW, Liu MT. Study of dental caries and correlated factors of 12-year-old children in Dongxiang, Baoan and Yugu races [In Chinese]. West China Journal of Stomatology 2009; 27(5):569-81.

34. Chu CH, Fung DS, Lo EC. Dental caries status of preschool children in Hong 
Kong. Br Dent J 1999; 187(11):616-20,605.

35. World Health Organization. Oral health surveys basic methods. 4th ed. Geneva: World Health Organization; 1997.

36. Chu CH, Chau AM, Wong ZS, Hui BS, Lo EC. Oral health status and behaviours of children in Myanmar - a pilot study in four villages in rural areas. Oral Health Prev Dent 2012; 10(4): 365-71.

37. Petersen PE, Kwan S, Zhu L, Zhang BX, Bian JY. Effective use of fluorides in the people's republic of china--a model for who mega country initiatives. Community Dental Health 2008; 25(4 Suppl 1):257-67.

38. Zhang QK, Li CW. Oral health status of Jinuo, Lahu, Yao ethnic minority school children in Xishuangbanna Prefecture [In Chinese]. Chinese Journal of Conservative Dentistry 1996; 6(4):245-6.

39. Lin HC, Schwarz E. Oral health and dental care in modern-day China. Community Dent Oral Epidemiol 2001; 29(5):319-28.

40. Rong WS, Bian JY, Wang WJ, Wang JD. Effectiveness of an oral health education and caries prevention program in kindergartens in China. Community Dent Oral Epidemiol 2003; 31(6):412-6.

41. Su RN, Wang RJ, Hong Y. Dental caries status among Wushengqi Mongolian ethnic minority middle school students in Yikezhaomeng, Inner Mongolia Autonomous District [In Chinese]. Journal of Stomatology 1996; 9(3):163.

42. Chu $\mathrm{CH}$, Lo ECM. Dental caries prevention and treatment for preschool children in China. Chin J Dent Res 2007; 10(Suppl):54-60.

43. Lu M, Hua S, Tao J. Dental caries status of 12 ethnic minority groups aged 3-6 in 16 regions in Yunnan Province [In Chinese]. Maternal and Child Health Care of China 2009; 24:100-1.

44. Zhu ZZ. Survey of dental caries among 0- to 12-year-old Miao ethnic minority children [In Chinese]. School Medicine 1988; 9(3):46.

45. Luo C. Epidemiology survey of dental caries of mongol children in high altitude regions[In Chinese]. Journal of High Altitude Medicine 2007; 17(4):50-1.

46. Wen SX, Zhou YL. Survey of 2- to 6-year-old children in high altitude region 
[In Chinese]. Chinese Journal of Conservative Dentistry 1999; 9(4):307.

47. Liu DG. Epidemiology survey of dental caries status among 1962 Tujia ethnic minority school students in Changyang [In Chinese]. Hubei Journal of Preventive Medicine 1996; 7(2):53.

48. Li ZY, Lian JY. Survey of dental caries status of Dongxiang primary and secondary school children [In Chinese]. Journal of Dental Prevention and Treatment 1998; 6(1):22.

49. $\mathrm{Xu}$ JH. Survey of dental caries status of adolescent in Jingji District, Wuzhong City [In Chinese]. Journal of Ningxia Medical College 1992; 14(2):77-9.

50. Zhang YW, Chen XL. Dental caries status of $3081 \mathrm{Li}$ ethnic minority people in Wuzhishan District, Hainan Province [In Chinese]. Journal of Clinical Stomatology 1992; 8(3):184-6.

51. Wang X, Chen G. Dental caries status of Lisu and Han adolescent in Nujiang Liuku region, Yunnan Province [In Chinese]. Journal of Modern Stomatology 1993; 7(2):99-100.

52. Luo MA, Yang GM. Dental caries of Naxi and Han adolescent in Ninlang region, Yunnan Province [In Chinese]. West China Journal of Stomatology 1983; 1(2):47-9.

53. Xue GC. Dental caries status of Tibetan and Han adolescent in Shannan region, Tibet [In Chinese]. Journal of Dental Prevention and Treatment 1995; 3(3):51-2.

54. Gao XQ, Huang KQ, Xi HJ. Investigation of dental caries status of permanent dentition amongst Tibetan students in Naqu, Tibet [In Chinese]. Chinese Journal of School Health 2007; 28(12):1097-8.

55. Liao ZM, Lu GH, Yan Y. Dental caries status of Tu and Han children in high altitude region, Qinhai Province [In Chinese]. Qinghai Medical Journal 1994; 142:4850.

56. Li JG, Cao H, Zhong CX. Dental caries status of 4020 Tujia ethnic minority school children in Hefeng County [In Chinese]. West China Journal of Stomatology 1994; 12(3):226-30.

57. Lin JH, Wang JH, Tian KC, Shu P, Xiang YC. Logistic regression analysis of multifactors on inducing dental caries among school children in Chongqing, China [In 
470 Chinese]. Modern Medicine Health 2003; 19(12):1519-1520.

471 58. Fen XL, Liu Y. Dental caries status of Uygur adolescent in Kashi region, 472 Xinjiang Province [In Chinese]. Journal of Clinical Stomatology 1996; 12(3):185-6.

473 59. Di L, Liu JB, A D, Hao YQ. Dental caries status of Uygur and Han children 474 aged 7- to 12 in Urumqi City [In Chinese]. Chinese Journal of School Doctor 1997; 475 11(5):379-80.

476 60. Fu KH, Zhao XF, Hong B. Oral health status of Yi ethnic minority shool children 477 in Hekou village, Xundian County [In Chinese]. Soft Science of Health 1999; 13(3):264787.

479 61. Huang SZ. Dental caries status of 1981 Zhuang ethnic minority school children 480 [In Chinese]. Chinese Journal of School Doctor 2002; 16(3):262-3. 